\title{
Universal stability curve for pattern formation in pulsed gas-solid fluidized beds of sandlike particles
}

\author{
Lilian de Martín, ${ }^{1,}{ }^{*}$ Coen Ottevanger, ${ }^{2}$ J. Ruud van Ommen, ${ }^{2}$ and Marc-Olivier Coppens ${ }^{1}$ \\ ${ }^{1}$ Department of Chemical Engineering, University College London, \\ Torrington Place, WC1E 7JE London, United Kingdom \\ ${ }^{2}$ Department of Chemical Engineering, Applied Sciences, Delft University of Technology, \\ Van der Maasweg 9, 2629 HZ Delft, The Netherlands
}

(Received 30 August 2017; published 8 March 2018)

\begin{abstract}
A granular layer can form regular patterns, such as squares, stripes, and hexagons, when it is fluidized with a pulsating gas flow. These structures are reminiscent of the well-known patterns found in granular layers excited through vibration, but, contrarily to them, they have been hardly explored since they were first discovered. In this work, we investigate experimentally the conditions leading to pattern formation in pulsed fluidized beds and the dimensionless numbers governing the phenomenon. We show that the onset to the instability is universal for Geldart B (sandlike) particles and governed by the hydrodynamical parameters $\Gamma=u_{\mathrm{a}} /\left(u_{\mathrm{t}} \bar{\phi}\right)$ and $f / f_{\mathrm{n}}$, where $u_{\mathrm{a}}$ and $f$ are the amplitude and frequency of the gas velocity, respectively, $u_{\mathrm{t}}$ is the terminal velocity of the particles, $\bar{\phi}$ is the average solids fraction, and $f_{\mathrm{n}}$ is the natural frequency of the bed. These findings suggest that patterns emerge as a result of a parametric resonance between the kinematic waves originating from the oscillating gas flow and the bulk dynamics. Particle friction plays virtually no role in the onset to pattern formation, but it is fundamental for pattern selection and stabilization.
\end{abstract}

DOI: 10.1103/PhysRevFluids.3.034303

\section{INTRODUCTION}

Granular flows are well known for exhibiting rich and complex mesoscale dynamics due to the self-organization of the composing grains. Examples include the ripples and dunes that spread over deserts and beaches, avalanches, viscous fingering, or patterns in rotating drums [1]. A phenomenon that has attracted great attention during the last two decades is the formation of standing wave patterns on the surface of vertically vibrated thin granular layers [2-4]. These patterns can be obtained in vacuo to avoid heaping and typically manifest themselves as stripes, squares, or hexagons, strongly resembling the structures formed in vertically vibrated fluids, also known as Faraday waves. Perhaps due to this analogy, vibrated granular patterns are often examined using the same control parameters as in the study of Faraday waves, i.e., the frequency $f$ and dimensionless acceleration $\Gamma=4 \pi^{2} f^{2} D / g$ of the vibrating plate, where $D$ is its displacement and $g$ is the gravitational acceleration. For viscous fluids, these driving parameters represent a balance between the energy injected and energy dissipated in the layer [5], and they can be derived from the Navier-Stokes equations governing the liquid flow.

\footnotetext{
*lilian.de.martin@chalmers.se
}

Published by the American Physical Society under the terms of the Creative Commons Attribution 4.0 International license. Further distribution of this work must maintain attribution to the author(s) and the published article's title, journal citation, and DOI. 


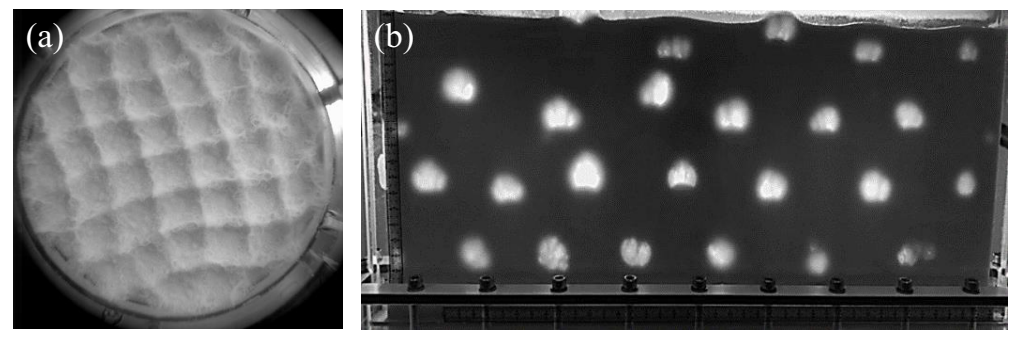

FIG. 1. Example of regular patterns in pulsed beds of Glass 240 particles: (a) square pattern in shallow $3 \mathrm{D}$ geometry, $h=3 \mathrm{~mm}, u=6.15+3.3[1+\sin (2 \pi 17 t)] \mathrm{cm} / \mathrm{s}$, and (b) triangular bubble tessellation in deep quasi-2D geometry [16], $h=15 \mathrm{~cm}$, bed thickness $=1 \mathrm{~cm}, u=1.4+8.3[1+\sin (2 \pi 5 t)] \mathrm{cm} / \mathrm{s}$.

However, continuum models that can adequately describe dense granular flows are lacking, so the physical interpretation of $\Gamma$ for granular patterns is less evident. Other control parameters, including the flying time of the layer and contact time with the vibrating plate, have been proposed in theoretical studies [6,7]; however, most experimental works define the parameter space using $f$ and $\Gamma$ due to their simplicity and straightforward calculation. The onset to the patterns exhibits a weak dependence on $f$ and occurs in the range $\Gamma \sim 2-3$ and $f \sim 7-100 \mathrm{~Hz}$, with a small decrease in $\Gamma$ for large frequencies $[4,8]$ and hysteresis at low frequencies, depending on whether the initial state is flat or patterned [9]. An additional control parameter is the ratio $h / d$, where $h$ is the layer height and $d$ is the particle diameter. Ratios $h / d \sim 3-30$ have been employed, with most results reported in the range 5-10.

Vibration is not the only periodic excitation that triggers the formation of standing wave patterns in granular layers. In 1999, Coppens and coworkers [10-12] showed experimentally that a granular bed fluidized with an oscillating gas flow can also form periodic patterns under certain conditions. In three-dimensional (3D) geometries, squares [Fig. 1(a)], stripes, and hexagons decorate the surface of the bed. For very shallow beds - a few mm deep — the dense phase remains bubbleless and particles oscillate coherently [13]. The patterns are subharmonic, meaning that the pattern is shifted by half a wavelength every pulse and the exact configuration is repeated after two pulses. If the thickness of the layer is increased, bubbles gradually form in alternating positions that match the pattern's antinodes, resulting in triangular bubble tessellations in the vertical plane for deep, quasi-two-dimensional (2D) geometries [Fig. 1(b)]. Although pure vibration can induce bubbling fluidization in deep beds $(h \sim$ $4 \mathrm{~cm}$ ) [14], such structured bubble arrays have not been reported in vibrated granular layers. It is unclear if this is due to physical reasons or experimental limitations. No discontinuities in the pattern parameters, such as the type of pattern and wavelength, are observed when transiting from the nonbubbling to the bubbling regime, indicating that regular patterns in shallow fluidized beds are the first manifestation of the same instability that develops into bubbles in deeper beds. In fact, bubbles in fluidized beds form in three stages [15]: (1) a one-dimensional (1D) traveling planar wave evolves from an unstable uniform stage, (2) this wave develops into a 2D transverse structure, and (3) particles are expelled through nonlinear mechanisms from a region with smaller-than-average particle concentration, forming a bubble. Regular patterns in shallow pulsed beds are the outcome of the secondary instability of the 1D planar voidage waves generated by the oscillating flow, and they can be used as a powerful tool to study this instability close to the onset, before it develops into bubbles.

Curiously, this phenomenon has been hardly explored since it was first observed, despite its potential to understand, control, and scale up fluidized beds [12]. In 2003, Li et al. [17] showed that the wavelength of fluidized patterns decreases with the flow frequency and follows a dispersion relation similar to the patterns formed in vibrated granular layers and liquids. At a computational level, Kawaguchi et al. [18] used Discrete Element Methods (DEM) in 2001 to study the behavior of pulsed fluidized beds. They reported that the use of pulsation frequencies of 4-5 Hz could induce some periodicity in the bubble dynamics for Geldart B particles. The computed pattern consisted of two large bubbles aligned horizontally and always nucleating at the same fixed positions. Another 
attempt to reproduce these patterns with DEM was made by Wang and Rhodes [19] in 2005. Some dynamical ordering was observed, but the clear regular structures formed in the experiments were missing. No further work was conducted until we recently revisited the topic. We showed that the classical implementation of two-fluid models fails to reproduce the experimental bubble patterns [20], as opposed to pattern formation in vertically vibrated granular layers in vacuo, which has been successfully described by continuous approaches [21]. This highlights the difficulty of modeling two-phase granular flow as compared to dry granular systems. It is important to comment here that two-fluid models can reproduce regular bubble patterns in pulsed fluidized beds after a careful choice of the parameters, but the bubbles always form in fixed positions. It is the subharmonic, alternating behavior of the experimental bubble patterns that has been impossible to reproduce so far with continuum descriptions. This is not a trivial detail, but one of the main manifestations of the underlying physics behind this phenomenon. Recently, we have reported the first successful simulation of pattern formation in pulsed beds by using DEM [16]. Our simulations show that patterns do not form in deep beds in the absence of particle friction, and that the subharmonic behavior is related to the compressive stress generated in the wake of bubbles in the plastic regime. This compressive stress is not correctly captured by the frictional closures used in two-fluid models, suggesting this reason for the failure of continuum simulations.

Despite the recent computational insights, the conditions under which patterns form, the dimensionless numbers governing the phenomenon, and the mechanisms that trigger the instability are still unknown. This work aims to shed light on these questions and discuss the differences between patterns in vibrated and pulsed layers in view of the results. In particular, we focus on fluidized beds that are a few mm deep. This is the simplest dynamic scenario because, under this condition, bubbles cannot evolve further from a wave state, so the nonlinear mechanisms that expel the particles from the more dilute regions, forming the bubble [15], are not expected to be dominant. Working close to the instability's onset facilitates the analytical study of the continuum equations behind this phenomenon, for example, by means of a linear stability analysis.

We show that the threshold for pattern formation in shallow pulsed beds is universal for Geldart B (sandlike) particles [22] and determined by the hydrodynamical parameters $\Gamma=u_{\mathrm{a}} /\left(u_{\mathrm{t}} \bar{\phi}\right)$ and $f / f_{\mathrm{n}}$, where $u_{\mathrm{a}}$ and $f$ are the amplitude and frequency of the gas velocity, respectively, $u_{\mathrm{t}}$ is the terminal velocity of the particles, $\bar{\phi}$ is the average solids fraction, and $f_{\mathrm{n}}$ is the natural frequency of the bed. Extensive experimentation supported by computational simulations shows that particle friction plays virtually no role in the onset to pattern formation, but it is fundamental for pattern selection and stabilization.

\section{EXPERIMENTAL}

Experiments were conducted in a cylindrical column $14 \mathrm{~cm}$ i.d. made of Perspex. The distributor is a 3-mm-thick porous plate Grade 07 from Sintertech (BK 10.30.07). More regular patterns are obtained with a distributor BK 10.30.10, but the amplitude of the sinusoidal gas velocity is strongly attenuated due to the higher pressure drop, complicating the operation at low values of the minimum gas flow. A granular layer of thickness $h$ is deposited inside the column and excited with a quasisinusoidal air flow fed through the distributor with superficial velocity $u=u_{\min }+u_{\mathrm{a}}[1+\sin (2 \pi f t)]$. This flow is realized by sending a sinusoidal voltage to a $154 \mathrm{~A}$ high-flow-control solenoid valve from MKS. It is noteworthy that there is no control loop, so the flow created by the valve is not perfectly sinusoidal. An empirical mode decomposition [23] reveals that the instantaneous frequency of the gas velocity fluctuates in time, instead of being constant as in a sinusoid. This fluctuation is approximately $\pm 1 \mathrm{~Hz}$ and increases when working at low values of $u_{\min }$ up to $\pm 2 \mathrm{~Hz}$. This is the reason why the setup is not operated at very low values of $u_{\min }$. The maximum flow rate for the solenoid valve is 200 standard liters per minute, which sets the maximum velocity amplitude $u_{\mathrm{a}}$ at $10-11 \mathrm{~cm} / \mathrm{s}$. The gas velocity is measured and recorded with a fast response flow-meter Omega FMA-1611 and evaluated based on the pressure drop across the distributor, after previous calibrations with constant gas flow. Both methods give comparable results. 
TABLE I. Geldart B particles used in the experiments. $u_{\mathrm{mf}}$ and $u_{\mathrm{t}}$ stand for minimum fluidization and terminal velocity, respectively.

\begin{tabular}{lcccc}
\hline \hline Name & $d_{\mathrm{p}}(\mu \mathrm{m})$ & $\rho_{\mathrm{p}}\left(\mathrm{kg} / \mathrm{m}^{3}\right)$ & $u_{\mathrm{mf}}(\mathrm{m} / \mathrm{s})$ & $u_{\mathrm{t}}(\mathrm{m} / \mathrm{s})$ \\
\hline Glass 130 & $130 \pm 20$ & 2500 & 0.012 & 0.80 \\
Glass 240 & $240 \pm 10$ & 2500 & 0.041 & 1.74 \\
Zinc 130 & $130 \pm 20$ & 7140 & 0.038 & 1.79 \\
Steel 130 & $130 \pm 20$ & 7900 & 0.042 & 1.93 \\
Polystyrene 600 & $600 \pm 100$ & 1040 & 0.115 & 2.49 \\
\hline \hline
\end{tabular}

Experiments have been conducted with five different kinds of carefully selected Geldart B particles [22] (Table I). Some of these particles are made of the same material and, therefore, share frictional parameters. Other ones have similar minimum fluidization and terminal velocity, presenting equivalent fluid-particle interactions. This strategy allows us to study the influence of particle friction and fluid-particle interactions independently.

By keeping $u_{\min }$ and $f$ constant, the amplitude of the gas velocity is slowly increased until the first undulations on the bed surface are observed at $u_{\mathrm{a}, \mathrm{c}}$. For each new value of the amplitude, the layer is excited for a few minutes to ensure that a lack of patterns is not due to transients. Similar to vibrated layers, the threshold for pattern formation is lower if the amplitude is decreased instead of increased, due to hysteresis. However, our goal is to study the onset to the instability from a flat, nonpatterned state and shed light onto the stabilizing and destabilizing mechanisms, so the instability chart corresponds to increasing flow amplitude. A discussion of the pattern wavelength and complete phase diagram is beyond the scope of this paper, but such a study of pattern formation has been conducted by Li et al. [17] for two types of particles.

\section{RESULTS AND DISCUSSION}

An example of the minimum gas velocity amplitude necessary to induce patterns, $u_{\mathrm{a}, \mathrm{c}}$, as a function of flow frequency $f$ and layer height $h$ is shown in Fig. 2. The instability threshold strongly depends on the flow frequency, decreasing with the frequency until reaching a plateau. An increase in $h$ displaces $u_{\mathrm{a}, \mathrm{c}}$ towards lower frequencies by an amount $\propto \sqrt{h}$, suggesting scaling with the natural frequency of the bed $f_{\mathrm{n}}$ [24]. In this work, $f_{\mathrm{n}}$ is estimated with the model of Baskakov

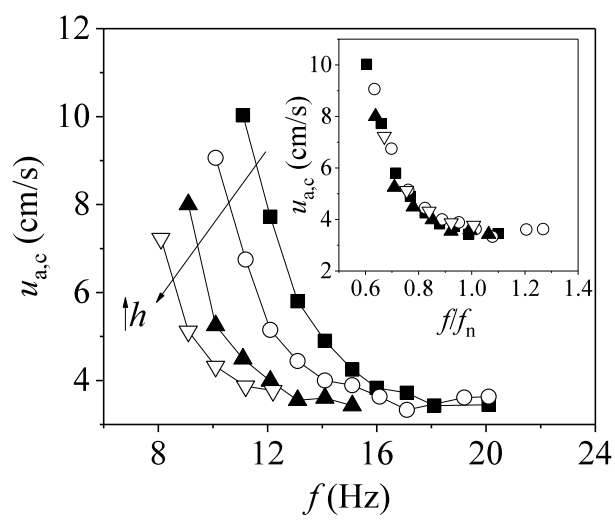

FIG. 2. Influence of the layer height $h$ on the threshold for pattern formation for Glass 240 particles at $u_{\min }=4 \mathrm{~cm} / \mathrm{s} . \nabla h=7 \mathrm{~mm}, \boldsymbol{\Delta} h=5 \mathrm{~mm}, \bigcirc h=4 \mathrm{~mm}, \boldsymbol{\nabla} h=3 \mathrm{~mm}$. (Inset) The curves collapse when $f$ is normalized by the natural frequency, $f_{\mathrm{n}}=\frac{1}{\pi} \sqrt{\frac{g}{h}}$, of the layer. 


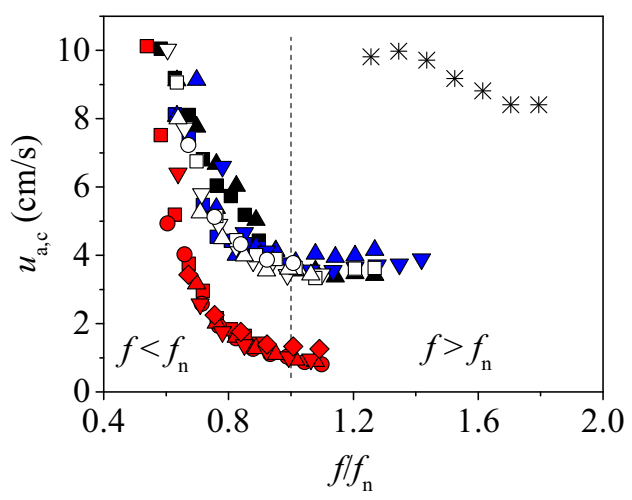

FIG. 3. Threshold for pattern formation for Glass 130 (red), Glass 240 (white), Zinc 130 (blue), Steel 130 (black), and Polystyrene 600 (asterisks). $u_{\min }=4 \mathrm{~cm} / \mathrm{s}$ for all cases and $h=2-8 \mathrm{~mm}$.

et al. [25] $f_{\mathrm{n}}=\frac{1}{\pi} \sqrt{\frac{g}{h}}$, which assumes that the bed oscillates like a liquid in a $\mathrm{U}$ tube. This equation gives comparable results to the model of Verloop and Heertjes [24,26], which was derived under assumptions that are closely related to our system, such as coherent particle motion and low bed height. This behavior is opposite to pattern formation in vibrated granular layers in vacuo, whose threshold shows a very low dependence on the vibration frequency and layer height. The role of the natural frequency here highlights one of the main differences between pulsed and vibrated beds: patterns in gas-fluidized beds can sustain an oscillation — as liquids do-whereas patterns in vibrated systems cannot. If the amplitude of the flow is suddenly stopped in a fluidized bed while keeping the average flow constant and above $u_{\mathrm{mf}}$, the subharmonic patterns persist with decaying amplitude. In contrast, if the excitation is stopped in a vibrated granular layer, patterns stop forming immediately. This corresponds to the liquidlike nature of fluidization. Indeed, if $f$ is normalized by $f_{\mathrm{n}}$, all curves collapse to a master curve centered around $f_{\mathrm{n}}$ (inset of Fig. 2).

Close inspection of the stability curves shown in Fig. 3 and the particle properties shown in Table I reveals that solids with similar $u_{\mathrm{t}}$ and $u_{\mathrm{mf}}$, that is, with similar fluid-particle interactions, share a stability curve, independently of the frictional properties of the particles. The amplitude of the gas flow that induces instability increases with the terminal velocity $u_{\mathrm{t}}$ or minimum fluidization velocity $u_{\mathrm{mf}}$ of the particles, indicating that $u_{\mathrm{mf}}$ or $u_{\mathrm{t}}$ have a stabilizing effect. Surprisingly, particle diameter and density do not have an isolated effect on the instability.

\section{Driving parameters and stability curve}

In contrast to vibration, which can be fully characterized in terms of frequency and amplitude, an oscillating gas flow has the minimum gas velocity $u_{\min }$, or equivalently, the average gas velocity $\bar{u}=u_{\min }+u_{\mathrm{a}}$, as an additional degree of freedom. Li et al. [17] suggest that $\bar{u}$ could be considered a control parameter similar to gravity in the expression $\Gamma=4 \pi^{2} f^{2} A / g$ used for vibrated patterns. Our experiments show that an increase in $u_{\text {min }}$ gradually decreases the threshold for pattern formation at high frequencies (Fig. 4). This change is very pronounced for Glass 240, Zinc 130, and Steel 130 when increasing $u_{\min }$ from 3 to $6 \mathrm{~cm} / \mathrm{s}$. In contrast, Glass 130 shows a small decrease for the same variation of $u_{\min }$. It is worth mentioning that Zinc 130, Steel 130, and Glass 240 have $u_{\mathrm{mf}} \sim 4 \mathrm{~cm} / \mathrm{s}$, so a change from $u_{\min }=3 \mathrm{~cm} / \mathrm{s}$ to $6 \mathrm{~cm} / \mathrm{s}$ can have a large effect in the bed dynamics because for $u_{\min }=3 \mathrm{~cm} / \mathrm{s}$, the system is defluidized at the minima of the gas flow, whereas for $u_{\min }=6 \mathrm{~cm} / \mathrm{s}$ the system is fully fluidized during the entire pulse cycle. On the other hand, Glass 130 has $u_{\mathrm{mf}} \sim 1.2 \mathrm{~cm} / \mathrm{s}$, so the bed is well fluidized during the entire pulse for both values of $u_{\min }$.

The effect of $u_{\min }$ can be corrected by taking into account how the average gas velocity $\bar{u}$ affects the average solids volume fraction $\bar{\phi}$. Actually, the stability curves can be collapsed onto a master 


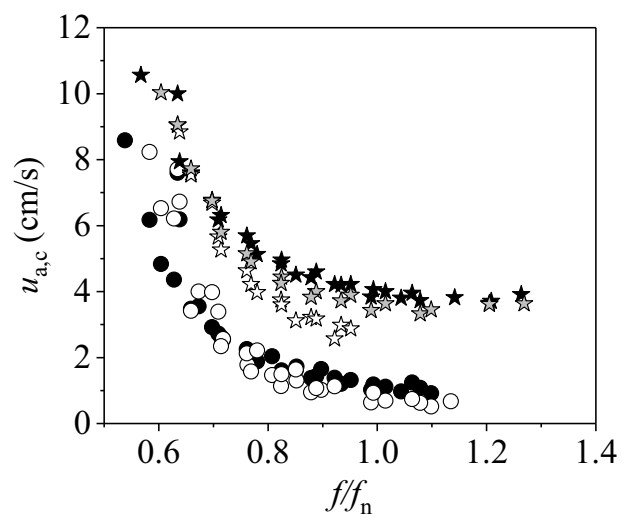

FIG. 4. Influence of $u_{\min }$ on the threshold for pattern formation for Glass 240 (stars) and Glass 130 (circles). Black, gray, and white symbols represent $u_{\min }=3,4$, and $6 \mathrm{~cm} / \mathrm{s}$, respectively.

curve when using the driving parameters $\Gamma=u_{\mathrm{a}} /\left(u_{\mathrm{t}} \bar{\phi}\right)$ and $f / f_{\mathrm{n}}$ (Fig. 5), where $\bar{\phi}$ is the solids volume fraction at $\bar{u}$, estimated from the Richardson-Zaki (RZ) equation $\bar{u} / u_{\mathrm{t}}=(1-\bar{\phi})^{n}$ with $(4.7-n) /(n-2.35)=0.175 \operatorname{Re}_{\mathrm{t}}^{0.75}[27]$ and $\operatorname{Re}_{\mathrm{t}}=d_{\mathrm{p}} \rho_{\mathrm{g}} u_{\mathrm{t}} / \mu_{\mathrm{g}}$.

The average solids volume fraction is determined with the RZ equation, instead of the experimental bed expansion, because the flanges that connect the windbox and freeboard in a typical fluidization setup impede any side visualization of the bed close to the distributor level. The expansion of a deeper bed cannot be used as a representative value either, because in a patterned shallow bed bubbles cannot evolve beyond the wave state. The bed is bubbleless, so all the gas flows through the dense phase, contributing to the expansion. If the bed height is increased, particles are expelled from the antinodes of the waves forming the bubbles, and a fraction of the gas will bypass the dense phase. In summary, for the same gas velocity, the solids volume fraction in a shallow patterned bed will be considerably smaller than in the dense phase of a deep, bubbling, bed of the same material. Therefore, we rely on the RZ equation to capture the power law dependence of the bed expansion with the gas velocity. Note that the RZ equation was derived for beds that expand homogeneously, an assumption that is satisfied for very shallow beds.

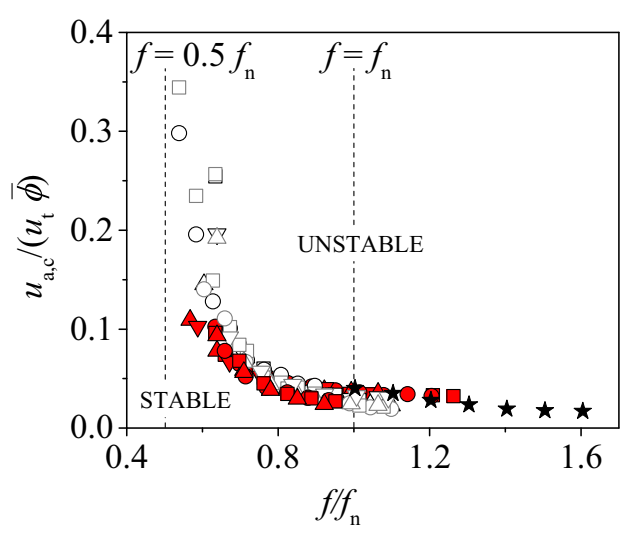

FIG. 5. Universal stability curve for pattern formation in pulsed fluidized beds of Geldart B particles. Plots include Glass 130 at $u_{\min }=3$ or $4 \mathrm{~cm} / \mathrm{s}$ and $h=2-7 \mathrm{~mm}$ (white symbols), Glass 240 at $u_{\min }=3$ or $6 \mathrm{~cm} / \mathrm{s}$ and $h=3-7 \mathrm{~mm}$ (red symbols), and Polysterene 600 at $u_{\min }=11.5 \mathrm{~cm} / \mathrm{s}$ and $h=10 \mathrm{~mm}$ (stars). For the sake of clarity, curves for Zinc 130 and Steel 130 are not plotted because they overlap with Glass 240 (see Fig. 3). 

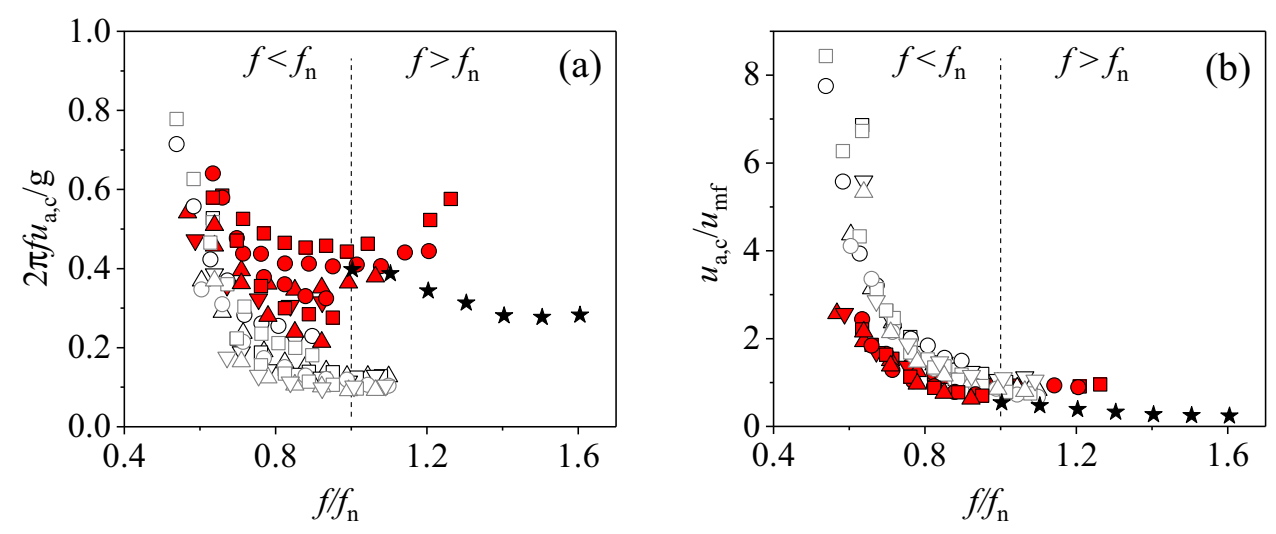

FIG. 6. Stability curves when the driving parameter is defined as (a) $\Gamma=2 \pi f u_{\mathrm{a}} / g$ and (b) $\Gamma=u_{\mathrm{a}} / u_{\mathrm{mf}}$. Symbols correspond to Fig. 5.

Although patterns can form for arbitrarily small $u_{\min }$, the solids volume fraction $\bar{\phi}$ estimated from the RZ equation represents only the average value when the bed is kept fluidized during the entire pulse cycle, that is, when $u_{\text {min }}>u_{\mathrm{mf}}$. In case the RZ equation predicts unrealistic values for $\bar{\phi}$ when working close to $u_{\mathrm{mf}}$, we impose that $\bar{\phi}$ cannot be larger than 0.58 , which is the experimental value determined for a randomly poured bed.

The driving parameter $\Gamma=2 \pi f u_{\mathrm{a}} / g$, which represents dimensionless acceleration and is analogous to $\Gamma=4 \pi^{2} f^{2} d / g$ used in vibrated granular layers and liquids, and vibrated-fluidized granular layers [28], leads to inconsistent results when applied to pulsed fluidized beds [Fig. 6(a)]. The driving parameter $\Gamma=u_{\mathrm{a}} / u_{\mathrm{mf}}$ used by Li et al. [17] to characterize patterns in pulsed fluidized beds leads to more coherent results [Fig. 6(b)]. These authors did not justify the choice of this parameter, but it contains the basic stabilizing and destabilizing mechanisms included in our driving parameter $\Gamma=u_{\mathrm{a}} /\left(u_{\mathrm{t}} \bar{\phi}\right)$. In fact, both $u_{\mathrm{mf}}$ and $u_{\mathrm{t}}$ emerge from a balance between drag and gravity, so it is expected that they generate similar curves. However, $\Gamma=u_{\mathrm{a}} / u_{\mathrm{mf}}$ produces significant discrepancies between different types of particles because it does not take into account the average solids volume fraction. Furthermore, $u_{\mathrm{mf}}$ represents the velocity at which the drag force exerted by the gas equals the bed weight; it is a parameter describing a static state with no apparent connection to the bed dynamics.

In contrast, both $u_{\mathrm{t}}$ and $\phi$ included in our definition of $\Gamma$ contribute to the formation of kinematic waves in fluidized beds, which are known to be instability drivers [29]. In particular, the role of these variables in the instability for pattern formation can be understood within the theoretical framework proposed by Batchelor [15]. Batchelor postulated that variations of disturbances in the vertical direction can be evened out by particle convection with velocity $V=|\phi d u / d \phi|_{\phi_{0}}$, where $u(\phi)=u_{\mathrm{t}}(1-\phi)^{n}$ is the mean fall velocity of particles and $\phi_{0}$ is the solids volume fraction in the uniform bed. That is, the rate at which particles fill a transverse disturbance in $\phi$ must be large compared to the growth rate of the disturbance and must increase with the magnitude of the disturbance. Taking as a reference the bed at $\bar{u}$, somehow, $\Gamma=u_{\mathrm{a}} /\left(u_{\mathrm{t}} \bar{\phi}\right)$ compares the maximum rate at which particles fill the growing transverse disturbance $\propto u_{\mathrm{t}} \bar{\phi}$ and the maximum growth rate of the disturbance $\propto u_{\mathrm{a}}$. Note that the hypothesis that the kinematic wave plays a role in pattern formation in fluidized beds does not contradict the fact that its velocity $u_{\mathrm{k}}=n u_{\mathrm{t}} \phi(1-\phi)^{(n-1)}$ [29] does not appear explicitly in the definition of $\Gamma$. In the field of pattern formation, the driving parameter compares the magnitude of two mechanisms, a destabilizing and a stabilizing one. For example, in vibrated layers, $\Gamma=4 \pi^{2} f^{2} D / g$ compares the maximum force exerted by the plate upwards and the maximum force bringing the layer downwards, which is gravity in the absence of interstitial fluid. In fluidized beds, $u_{\mathrm{a}}$ and $u_{\mathrm{t}}$ can be related to the maximum upwards and downwards drag force. It is 
possible that the parameter $\Gamma$ leading to perfect collapse of the threshold curves is a more elaborate version of $\Gamma=u_{\mathrm{a}} /\left(u_{\mathrm{t}} \bar{\phi}\right)$, which is not taking into account the variation of the drag coefficient with the Reynolds number, at least explicitly. However, the fact that $\Gamma=u_{\mathrm{a}} /\left(u_{\mathrm{t}} \bar{\phi}\right)$ provides that good overlap for such different solids indicates that this parameter captures the main features of the phenomenon, and it can be used as a basis to further study these patterns.

Patterns form for flow frequencies in the vicinity of the natural frequency of the bed, regardless of particle properties. The threshold dramatically increases when approaching $f / f_{\mathrm{n}} \sim 0.5$, setting the lower frequency boundary for pattern formation. This ratio corresponds to the principal subharmonic component of parametric resonance in some systems [30], so it is possible that a singularity appears around this value. The connection between this divergence and the physics of pulsed granular matter is something that deserves further attention. The threshold decreases with the frequency of the gas velocity and reaches a plateau at $f_{\mathrm{n}}$, suggesting parametric resonance between the kinematic waves originating from the oscillating flow, characterized by $u_{\mathrm{t}}$ and $\phi$ and the bulk dynamics, characterized by $f_{\mathrm{n}}$. Researchers investigating pulsation in nonpatterned fluidized beds as a means to intensify fluidization also find that the largest effects are observed in the range $2-10 \mathrm{~Hz}$, matching the natural frequency of typical laboratory-scale fluidized beds [31]. If patterned and nonpatterned pulsed beds share dynamical features, the study of general, pulsed fluidization could greatly benefit from the study of these patterns. For example, one of the main conclusions about nonpatterned pulsed beds exposed in a recent review [31] is that no one has been able to highlight the relationship between pulsed flow and particle characteristics. The parameters $\Gamma=u_{\mathrm{a}} /\left(u_{\mathrm{t}} \bar{\phi}\right)$ and $f / f_{\mathrm{n}}$, and the results shown in Fig. 5 might be a first fundamental step towards this.

The natural frequency of the bed is of fundamental importance if one is to structure deep fluidized bed reactors by using regular patterns [12]. The deeper the bed, the lower the frequency of the flow must be to trigger the patterns. However, the lower the frequency, the larger the wavelength of the patterns becomes $[11,17]$. This means that patterns in deep beds form only if one of the horizontal dimensions of the bed is large enough to fit the pattern, that is, equal to the pattern wavelength at the very least. Actually, to study these patterns from a fundamental point of view in the absence of wall effects, this ratio should be much larger [32].

The normalized curves show little dependence on the particle surface (Fig. 5), suggesting that friction plays a minor or no role triggering the patterns. This is also the case for patterns formed in vibrated granular layers, in which the instability is usually explained in terms of flying time of the granular layer, layer dilation, and layer acceleration at the moment of collision with the plate. Moon et al. [33] used molecular dynamics to show that frictionless particles can also form patterns, and that the threshold for pattern formation is only $30 \%$ lower than the threshold for particles with friction. However, only striped patterns were stable for frictionless particles due to a larger grain mobility and lower overall collision rate. The role of friction in pattern formation in pulsed fluidized beds and its implications in continuum modeling of fluidization is an interesting topic that will be studied in more detail in upcoming publications.

\section{CONCLUSIONS}

The mechanism behind the instability leading to pattern formation in pulsed gas-fluidized and vertically vibrated granular layers is completely different as a result of the forces induced by the percolating gas phase. We show that the onset to pattern formation in shallow, bubbleless, pulsed fluidized beds of Geldart B particles is universal and governed by the parameters $\Gamma=u_{\mathrm{a}} /\left(u_{\mathrm{t}} \bar{\phi}\right)$ and $f / f_{\mathrm{n}}$, being virtually independent of friction. Patterns form in the vicinity of the natural frequency of the bed, possibly as a result of a parametric resonance between the kinematic waves originated by the oscillating gas and the bulk dynamics. Although the physics behind this complex phenomenon are still unclear, the definition of these control parameters represents a first step towards a fundamental understanding of fluidization and can be used as a basis to unravel the relationship between particle and flow properties in conventional pulsed beds. 
Industrial fluidized beds are deeper than the beds investigated in this work. However, the shallow bed limit represents the simplest dynamic scenario, because the instability that forms the bubbles is close to the onset. No bubbles are formed, only waves, so the nonlinear mechanisms responsible for bubble formation are not expected to be dominant. Although the stability curve for pattern formation in deep beds might be different from that for shallow beds, the results and insights derived from our work could be used as a basis to understand more complex scenarios.

\section{ACKNOWLEDGMENTS}

The authors gratefully acknowledge $\mathrm{K}$. Wu and M. Talebi for their contributions to the experiments. The research leading to these results has received funding from an EPSRC Frontier Engineering Award (EP/K038656/1).

[1] I. S. Aranson and L. S. Tsimring, Patterns and collective behavior in granular media: Theoretical concepts, Rev. Mod. Phys. 78, 641 (2006).

[2] S. Fauve, S. Douady, and C. Laroche, Collective behaviors of granular masses under vertical vibration, J. Phys. Colloques 50, C3-187 (1989).

[3] F. Melo, P. B. Umbanhowar, and H. L. Swinney, Transition to Parametric Wave Patterns in a Vertically Oscillated Granular Layer, Phys. Rev. Lett. 72, 172 (1994).

[4] F. Melo, P. B. Umbanhowar, and H. L. Swinney, Hexagons, Kinks, and Disorder in Oscillated Granular Layers, Phys. Rev. Lett. 75, 3838 (1995).

[5] E. A. Cerda and E. L. Tirapegui, Faraday's instability in viscous fluid, J. Fluid Mech. 368, 195 (1998).

[6] E. Cerda, F. Melo, and S. Rica, Model for Subharmonic Waves in Granular Materials, Phys. Rev. Lett. 79, 4570 (1997).

[7] C. Bizon, M. D. Shattuck, and J. B. Swift, Linear stability analysis of a vertically oscillated granular layer, Phys. Rev. E 60, 7210 (1999).

[8] T. H. Metcalf, J. B. Knight, and H. M. Jaeger, Standing wave patterns in shallow beds of vibrated granular material, Physica A 236, 202 (1997).

[9] P. B. Umbanhowar, F. Melo, and H. L. Swinney, Periodic, aperiodic, and transient patterns in vibrated granular layers, Physica A 249, 1 (1998).

[10] Y. Cheng, S. Kaart, C. M. van den Bleek, and M.-O. Coppens, Control of chaotic dynamics in a 2D fluidized bed by periodic gas injection, in Proc. AIChE Annual Meeting, Vol. 31, edited by L Glicksman (AIChE, Dallas, Texas, USA, 1999), pp. 312-319.

[11] M.-O. Coppens, M. A. Regelink, and C. M. van den Bleek, Pulsation induced transition from chaos to periodically ordered patterns in fluidised beds, in Proc. 4th World Conf. on Particle Technology (WCPT2002) (IEAust, Sydney, Australia, 2002), paper 355, pp. 1-8.

[12] M.-O. Coppens and J. R. van Ommen, Structuring chaotic fluidized beds, Chem. Eng. J. 96, 117 (2003).

[13] See Supplemental Material at http://link.aps.org/supplemental/10.1103/PhysRevFluids.3.034303 for a slow motion video of a 3D square pattern.

[14] H. K. Pak and P. R. Behringer, Bubbling in vertically vibrated granular materials, Nature (London) 371, 231 (1994).

[15] G. K. Batchelor, Secondary instability of a gas-fluidized bed, J. Fluid. Mech. 257, 359 (1993).

[16] K. Wu, L. de Martín, and M.-O. Coppens, Pattern formation in pulsed gas-solid fluidized beds - The role of granular solid mechanics, Chem. Eng. J. 329, 4 (2017).

[17] J. Li, I. S. Aranson, W.-K. Kwok, and L. S. Tsimring, Periodic and Disordered Structures in a Modulated Gas-Driven Granular Layer, Phys. Rev. Lett. 90, 134301 (2003).

[18] T. Kawaguchi, A. Miyoshi, T. Tanaka, and Y. Tsuji, Discrete particle analysis of 2D pulsating fluidized bed, in Proc. 4th Intl. Conf. on Multiphase Flow (ICMF-2001), New Orleans, USA (2001), paper 838. 
[19] X. S. Wang and M. J. Rhodes, Pulsed fluidization—A DEM study of a fascinating phenomenon, Powder Technol. 159, 142 (2005).

[20] K. Wu, L. de Martín, L. Mazzei, and M.-O. Coppens, Pattern formation in fluidized beds as a tool for model validation: A two-fluid model based study, Powder Technol. 295, 35 (2016).

[21] J. Bougie and K. Duckert, Continuum simulations of shocks and patterns in vertically oscillated granular layers, Phys. Rev. E 83, 011303 (2011).

[22] D. Geldart, Types of gas fluidization, Powder Technol. 7, 285 (1973).

[23] N. E. Huang, Z. Wu, S. R. Long, K. C. Arnold, X. Chen, and K. Blank, On instantanous frequency, Adv. Adapt. Data Anal. 01, 177 (2009).

[24] H. T. Bi, A critical review of the complex pressure fluctuation phenomenon in gas-solids fluidized beds, Chem. Eng. Sci. 62, 3473 (2007).

[25] A. P. Baskakov, V. G. Tuponogov, and N. F. Filippovsky, A study of pressure fluctuations in a bubbling fluidized bed, Powder Technol. 45, 113 (1986).

[26] J. Verloop and P. M. Heertjes, Periodic pressure fluctuations in fluidized beds, Chem. Eng. Sci. 29, 1035 (1974).

[27] P. N. Rowe, A convenient empirical equation for estimation of the Richardson-Zaki exponent, Chem. Eng. Sci. 42, 2795 (1987).

[28] E. Cano-Pleite, F. Hernández-Jiménez, and A. Acosta-Iborra, Bulk oscillation and velocity wave propagation in a vibrated fluidized bed at minimum fluidization conditions, Powder Technol. 308, 346 (2017).

[29] L. Gibilaro, Fluidization-Dynamics (Butterworth-Heinemann, Oxford, 2001).

[30] A. Champneys, in Mathematics of Complexity and Dynamical Systems, edited by R. A. Meyers (Springer, New York, 2011), p. 183.

[31] E. Ireland, K. Pitt, and R. Smith, A review of pulsed flor fluidization; the effects of intermittent gas flow on fluidised gas-flow bed behaviour, Powder Technol. 292, 108 (2016).

[32] M. Cross and H. Greenside, in Pattern Formation and Dynamics in Nonequilibrium Systems, edited by M. Cross and H. Greenside (Cambridge University Press, Cambridge, 2009).

[33] S. J. Moon, J. B. Swift, and H. L. Swinney, Role of friction in pattern formation in oscillated granular layers, Phys. Rev. E 69, 031301 (2004). 